\title{
La ville intelligente et la question de la participation citoyenne dans les collectivités publiques
}

The intelligent city and the question of citizen participation in public authorities.

Die intelligente stadt und die frage der beteiligung der bürger an den öffentlichen behörden.

\section{Adèhè Essossimna POKORE}

\section{(2) OpenEdition}

\section{Journals}

Édition électronique

URL : http://journals.openedition.org/ctd/2958

DOI : $10.4000 /$ ctd. 2958

ISSN : 2491-1437

\section{Éditeur}

Chaire Unesco Pratiques émergentes en technologies et communication pour le développement

\section{Édition imprimée}

ISBN : 2491-1437

Référence électronique

Adèhè Essossimna POKORE, « La ville intelligente et la question de la participation citoyenne dans les collectivités publiques ", Communication, technologies et développement [En ligne], 8 | 2020, mis en ligne le 09 août 2020, consulté le 28 mars 2021. URL : http://journals.openedition.org/ctd/2958 ; DOI : https://doi.org/10.4000/ctd.2958

Ce document a été généré automatiquement le 28 mars 2021.

Communication, technologies et développement 


\section{La ville intelligente et la question de la participation citoyenne dans les collectivités publiques}

The intelligent city and the question of citizen participation in public authorities.

Die intelligente stadt und die frage der beteiligung der bürger an den

öffentlichen behörden.

Adèhè Essossimna POKORE

1 La ville intelligente constitue un dispositif de participation citoyenne dans les collectivités publiques. En effet, elle offre la possibilité aux citoyens de participer à la prise de décision concernant les actions publiques par le biais des Nouvelles Technologies de l'Information et de la Communication (NTIC). Toutefois, un constat peut être fait, le débat qui précède la construction de ladite ville n'est pas inclusif. Les habitants ne sont pas impliqués dans ce débat. De fait, le mécanisme de la mise en place de la ville intelligente repose uniquement sur la vision des spécialistes ou experts. Ce qui justifie quelquefois qu'une ville intelligente mise en place par une collectivité publique donnée ne corresponde pas aux aspirations des habitants. De ce fait, ici, nous estimons que le manque de la participation des citoyennes au débat précédant la construction de ladite ville constitue la source de la vulnérabilité des habitants. À cet effet, nous suggérons que ce débat soit inclusif. Il s'agit d'associer les citoyens afin qu'ils aient la possibilité de décider de la forme de la ville intelligente qui les convient. Ce qui permettrait de trouver une solution à la question suivante : quelle forme de ville intelligente pour quelle population? Si non, une collectivité publique peut concevoir une ville intelligente au profit de ses habitants sans que celle-ci ne corresponde réellement à leurs aspirations, comme c'est le cas de la cité numérique construite en Algérie. En effet (Abderezak Djemili et Massaoud Abbaoui, 66), indiquent que : « [...] La cité numérique reste encore largement "une boîte noire", pour les habitants, sous l'effet d'une double fermeture: corporatiste et administrative. ". Conséquence, la cité numérique ne correspond pas aux habitudes des habitants. Ils préconisent donc une participation des 
citoyens dans la construction et la gestion de la cité numérique. Nous estimons dans cette perspective qu'une ville intelligente ne doit pas seulement renforcer les principes démocratiques en aval, elle doit, elle-même, être construite sur la base de la «technologie participative » en amont.

2 À cet effet, dans cet article, d'abord, nous présenterons la ville intelligente, les avantages qu'elle procure en matière du renforcement des pratiques démocratiques et le concept de participation citoyenne. Ensuite, nous relèverons, dans un esprit critique, le manque de la participation citoyenne dans le débat qui précède la conception et la construction de ladite ville. Enfin, nous envisagerons des dispositifs susceptibles de favoriser la participation des habitants au débat qui précède la construction de la ville intelligente afin d'éviter la vulnérabilité de ces derniers.

\section{La « ville intelligente » : émergence, spécificité et avantages}

3 La ville intelligente tire ses origines de la philosophie prônée par le mouvement « open data ${ }^{1}$ ", lequel tire ses origines du rêve de villes totalement connectées, que (Nicolas Schöffer, 91) qualifie de "ville informative ». De fait, le mouvement « open data » œuvre en faveur des principes d'échange égalitaire, de circulation libre et gratuite des données publiques. En réalité, lorsque les données publiques sont accessibles et réutilisables, elles acquièrent de nouvelles valeurs. De ce fait, il est souhaitable que des mécanismes favorisant la libre circulation de ces données soient mis en place. C'est dans cette perspective qu'il a été envisagé un espace urbain construit sur la base d'intégration des NTIC. Par contre, certains, à l'instar de Fabien Deglise, considèrent que ce projet de ville intelligente est utopiste. Cependant, le rêve est devenu une réalité, car de nos jours, la ville intelligente constitue le modèle urbain dans le monde entier, comme l'affirme (Laetitia Anthoine Gazel, 4) «si jusque récemment la smart city n'était qu'un concept, aujourd'hui c'est un projet que chaque ville désire mettre en place.». En effet, cette forme de ville portée dans les idées s'est concrétisée en 2003 en Corée du Sud, avec le projet U-Korea, lequel, en s'appuyant sur le concept d'«ubiquitous computing» de l'aménagement de la ville Songdo située au bord de la mer Jaune, a abouti à la mise en place des systèmes intelligents innovants notamment la gestion de l'énergie, le maillage Wifi et la vidéo surveillance omniprésente du réseau omniprésent à la société connectée. Cependant, c'est en 2005 que le concept de "smart city" a fait son apparition. Il a été utilisé par Bill clinton lors d'un défi lancé par sa fondation à l'entreprise Cisco qui avait la responsabilité de mettre en place des plans de décongestion des villes de Francisco, de Séoul et d'Amsterdam. Cette initiative visait à diminuer les émissions de $\mathrm{CO} 2$, réduire la pollution de l'environnement et de permettre aux collectivités locales et leurs habitants d'économiser le temps de leurs trajets et l'argent en réduisant leurs coûts budgétaires. À ce propos, Benoit Georges affirme que "lancé il y a moins de dix ans en Californie par une trentaine de pionniers de l'Internet, dont le célèbre professeur de droit Lawrence Lessig, le mouvement en faveur de l'ouverture des données publiques ("open data») s'est répandu sur tous les continents².».

4 De nos jours, ce mouvement a un statut important sur le plan mondial, ce qui fait affirmer (Julien Damon, 170) que : "c'est un qualificatif à la mode ces temps-ci.». Dans cette perspective, il a été mis en place depuis 2014 la norme ISO 37120 qui certifie les indicateurs sur les services urbains, la qualité de vie et la protection de 
l'environnement. Le forum, Intelligent Community Forum³, est organisé toutes les années au cours duquel les villes sont récompensées à titre des efforts consentis en matière de politique d'open data. La ville intelligente représente désormais le modèle de référence des municipalités dans le monde entier. C'est le cas de la ville de Nantes (France) qui fait des efforts afin de se hisser au rang de ville intelligente au plan national et international. Nous pouvons donc estimer que l'action sur la ville est désormais envisagée dans une logique plus qualitative de renouvellement.

Il convient de souligner que la révolution numérique favorise la naissance des villes connectées. Ces villes offrent plusieurs possibilités aux habitants dans les collectivités publiques. Pour (Julien Damon, 170), " une ville est plus ou moins intelligente si elle favorise le bien-être de ses habitants en leur donnant facilement accès aux ressources numériques. Je pense qu'une ville intelligente est d'abord une ville qui propose $d u$ wi-fi gratuit à tout le monde.". La ville intelligente joue un rôle important de nos jours en matière de la qualité sociale et environnementale par le biais des NTIC. En réalité, elle constitue une solution fiable et durable aux problèmes urbains contemporains. Par exemple, comme l'a montré (Jean Bouinot, 15), une ville intelligente est, non seulement, celle qui attire les entreprises, mais elle est aussi, celle qui est capable de retenir celles qui emploient la main-d'œuvre qualifiée. Elles constituent donc une solution au paradoxe que soulève l'explosion démographique urbaine. En réalité, dans les pays développés, la maind'œuvre qualifiée est devenue, malgré une population importante urbaine, une ressource plus rare que les capitaux financiers et des équipes actives stratégiques fondamentales dans un contexte mondial concurrentiel. Dès lors, la question qui se pose est de savoir quelle est la spécificité de la ville intelligente?

\section{La spécificité de la ville intelligente}

6 Une ville intelligente est principalement connectée en interne ${ }^{4}$ et en externe ${ }^{5}$. Elle est un système essentiellement complexe, mais totalement unifié. Même si elle est plus complexe, elle est de même nature que le schéma de la ville présenté par Nicolas Schöffer en 1974. Considérant ce schéma, nous pouvons admettre qu'une ville intelligente est comparable à un corps humain composé de plusieurs organes. Chaque organe joue un rôle spécifique en participant au bon fonctionnement de l'ensemble de l'organisme, dont les NTIC, contrôlées par un « centre cybernétique », représentent le cerveau humain. Cette forme de ville se présente, selon (Jean Daniélou, 63), «[...] comme un système unifié où les silos, souvent considérés comme autonomes et déconnectés les uns des autres, sont réunis par un réseau rassemblant des données éparses pour les traiter comme un tout.» La connexion en interne et en externe constitue donc la spécificité de la ville intelligente. Quelle peut-être alors l'importance de ces deux formes de connexion?

\section{Une ville intelligente, levier d'ouverture et de disponibilité des données publiques}

7 Les connexions interne et externe de la ville intelligente favorisent l'échange égalitaire, la circulation libre et gratuite des données dans les collectivités publiques. Sur le plan intérieur, par le biais des NTIC (les NTIC domestiques, supports, numériques, dispositifs d'informations, capteurs, compteurs intelligents, etc.), un secteur A peut mettre ses données collectées à la disposition d'un autre secteur $\mathrm{B}$, vice versa. C'est cette 
interconnectivité qui fait dire à (Herbert Maisl, 15) que «les Échanges de Données Informatisées vont en se multipliant à l'intérieur de l'administration comme dans les relations de celle-ci avec les entreprises. » Par exemple, les banques sollicitent les données judiciaires de leurs clients lors d'octroi des prêts et de crédits. Le secteur de transport met les données concernant tout son trafic à la disposition publique. Sur le plan extérieur, les informations circulent entre les villes ou entre les États par le biais des NTIC. De nos jours, face aux menaces du terrorisme islamiste auxquelles s'exposent tous les pays, nous assistons à une forte collaboration entre les États qui se traduit par la transmission des informations via la connectivité des villes. Par exemple, le terroriste français le plus recherché depuis 2015, Peter Cherif, a été arrêté en Djibouti le 16 décembre 2018 grâce à l'échange d'informations entre la France et le Djibouti.

Ainsi pouvons-nous admettre que les connexions interne et externe favorisent la disponibilité et la libre circulation des données publiques dans les collectivités publiques. En quoi la ville intelligente peut-elle alors garantir la pratique participative ? Avant de répondre à la question ainsi posée, nous présentons d'abord le concept de participation citoyenne.

\section{La participation citoyenne, origine et définition}

9 Le besoin d'avoir recours à la pratique participative dans le processus de prise de décisions politiques tire ses origines de l'Antiquité grecque dans la cité athénienne ${ }^{6}$. En effet, dans cette cité, les citoyens se regroupaient régulièrement sur la place publique, débâtaient puis décidaient et adoptaient ensemble des lois dans l'intérêt public. La responsabilité publique était attribuée aux citoyens lors de ces assemblées et elle pouvait faire l'objet de révocation de la même façon. C'est la marque de la démocratie directe, instaurée dans ladite cité à l'issue des réformes politiques et sociales réalisées par des hommes politiques ${ }^{7}$ suite à une crise politique et sociale. Cette forme de démocratie peut être considérée comme l'origine lointaine de la pratique participative. Par contre, l'impératif participatif, de nos jours, peut être expliqué par la crise de la représentativité à laquelle font face les démocraties représentatives et qui se manifeste par la « revendication de la démocratie ${ }^{8}$.». En effet, selon l'opinion publique, les représentants jouissent d'une liberté totale vis-à-vis des représentés, car les représentés n'ont qu'un seul pouvoir, l'élection de leurs gouvernants. Dans ces conditions, c'est la transparence administrative qui fait défaut. Dès lors, la pratique participative favorise la lisibilité et la visibilité de l'administration publique. Pour (Pierre Rosavanllon, 242), il s'agit, non seulement, de favoriser une meilleure compréhension du fonctionnement des institutions publiques, mais aussi, de mettre en place des mécanismes susceptibles de favoriser la bonne compréhension et l'interprétation du monde social. La pratique participative se conçoit donc comme une gestion collaborative des affaires publiques. Il est impératif d'associer les citoyens à la prise des décisions dans l'accomplissement des actions publiques. Il s'agit d'impliquer efficacement les citoyens et de tenir compte de leurs avis dans le processus de détermination et de réalisation des projets à intérêt public. Dans cette perspective (Marie-Hélène Bacqué et Yves Sintomer, 10) indiquent que certains auteurs, à l'instar de Patman, Mollet, Gontcharoff, Godbout, MacPherson, Barber, etc., ont commencé à défricher cette question participative dans les années 1970 et 1980 dans un contexte de développement des mouvements sociaux avant qu'elle ne devienne une question à 
portée mondiale. À ce propos (Pierre Rosanvallon, 242) indique que la pratique participative a d'abord commencé dans l'ordre économique sous forme d'une exigence du droit de savoir des citoyens et elle s'est ensuite développée dans plusieurs domaines. Par exemple, dans les politiques scientifiques, commencée dans les années 1960, c'est en 1970 qu'elle est abordée de façon systématique. Elle consiste à avoir recours au principe de précaution?.

10 Il paraît donc légitime d'estimer que la pratique participative consiste, non seulement, à impliquer toutes les parties prenantes dans le processus de prise de décision, mais aussi, la prise en compte de leurs différents avis s'avère obligatoire. Dans cette perspective, la théorie de l'enquête de John Dewey présente le moyen pratique par lequel les membres d'une communauté donnée peuvent parvenir ensemble à trouver des solutions aux maux qui minent leur société. À ce titre (John Dewey, 169) a présenté la définition de l'enquête relevant la nécessité d'impliquer toutes les parties prenantes dans le processus de prise de décisions. Nous pouvons admettre avec (Albert Ogien et Sandra Laugier, 106) que "[...] tous les membres d'une société se trouvent à égalité de responsabilité et de compétence dans le travail collectif qui consiste à s'occuper des questions d'intérêt public qui se posent incessamment à eux [...]》. Dès lors, si nous considérons la théorie de l'enquête, pourrions-nous admettre que le modèle de la ville de Songdo ${ }^{10} a$ été conçu et adopté dans un contexte de participation citoyenne?

\section{La conception et la construction de la ville intelligente, une affaire des experts et spécialistes}

11 La ville intelligente est susceptible de favoriser la transparence administrative, car elle est le canal de la mise à disposition des données relatives aux actions publiques des représentants. Cependant, le constat qui peut être fait est le suivant: la ville intelligente constitue un facteur de la participation citoyenne lorsqu'elle est fonctionnelle. En réalité, les conditions de sa conception et de sa construction ne font pas l'objet de transparence. En nous référant aux origines de ladite ville présentées par (Sandra Breux et Jérémy Diaz, 2), nous pouvons admettre que ce sont les experts et spécialistes qui décident des formes de villes intelligentes pour les habitants. De fait, le modèle de la ville intelligente a été une initiative des entreprises privées à l'instar de l'entreprise Cisco. Portée d'abord dans les idées, la ville intelligente a été concrétisée en Corée du sud en 2003 pour la première fois. Cette forme de ville s'est développée dans le monde entier. Dans ces conditions, il paraît légitime d'admettre que les habitants n'ont pas été impliqués dans le débat précédant la construction de la ville intelligente. (Abderezak Djemili et Messaoud Abbaoui, 66) soulignent justement qu'en Algérie, les cités numériques ont été construites sans consulter les habitants. En effet, ces cités regroupent un ensemble de logements collectifs $(20,60,150,400,6000$, etc.) construits en dehors des centres villes mais qui ne correspondent pas aux attentes des habitants. Nous pouvons donc admettre avec (Abderezak Djemili et Messaoud Abbaoui, 66) que la cité numérique "représente une organisation basée sur le quantitatif». Par contre, nous pouvons soutenir qu'afin que la ville intelligente soit basée sur le qualitatif, il faudrait mettre en place des dispositifs et procédures qui permettent d'associer les habitants au débat qui précède sa construction. Comment peut-on alors associer les habitants au débat précédant la conception et la construction de leur ville intelligente? 


\section{La participation citoyenne, fondement de la conception et la construction de la ville intelligente}

12 La mise en place d'une ville intelligente implique la modification du mode de vie des populations locales, car à la différence de la ville classique, elle suppose l'intégration des NTIC à l'espace urbain. De ce fait, si cette ville ne correspond pas aux réalités quotidiennes des habitants, ceux-ci se retrouveraient dans un état de vulnérabilité, car ils ne sauront pas se servir des dispositifs technologiques. Pour cette raison, l'association des habitants au débat qui précède la construction de la ville intelligente s'avère indispensable comme le suggère (Pascale Luciani-Boyer, 79)

«Lorsque l'on parle de la ville intelligente, on parle d'une ville qui se tisse. La ville est un grand métier à tisser, or quand on tisse une toile avec un métier, il y a des fils horizontaux et verticaux et chaque nœud est un lieu d'échange. Je crois que la ville intelligente, si on voulait y mettre une image, est ce merveilleux tissage que nous devons, politiques, mais pas exclusivement, avec aussi citoyens et société civile, construire.»

13 En réalité, une participation citoyenne peut permettre aux responsables des collectivités publiques de marier la rationalité technique de la ville intelligente avec les logiques et réalités des habitants. (Pascale Luciani-Boyer, 83) souligne justement que :

« [...], ma petite place, plutôt de comporter deux lampadaires, devrait compter un banc et trois pots de fleures. La puissance publique n'en sait rien, le décideur public n'en sait rien. Ceux qui le savent, ce sont bien les citoyens qui sont au plus près de leurs attentes quotidiennes. "

C'est ce qui constitue, à notre avis, l'importance de l'association des habitants au débat précédant la conception et la construction de la ville intelligente. Dès lors, comment pouvoir associer les habitants au débat qui précède la construction de la ville intelligente?

\section{L'« enquête », fondement de la conception et la construction de la ville intelligente}

Pour (John Dewey, 211-212), certaines questions peuvent être confiées aux experts ou spécialistes et faire toujours l'objet d'enquête. Il indique donc que, lorsqu'une question est confiée aux experts ou spécialistes qui font des recherches et qui aboutissent aux résultats, quels qu'ils soient, ces résultats doivent faire l'objet d'un examen public. Ils doivent impérativement être portés à la connaissance des autres membres de la société afin qu'ils fassent un examen critique. Nous pouvons donc estimer que, pour John Dewey, le plus important dans la gestion des affaires publiques c'est de permettre à tous les membres de la société de porter un regard critique sur les propositions de décisions qui sont envisagées. Si les experts ou spécialistes ont l'obligation de mettre les résultats de leurs investigations à la disposition publique, leurs résultats peuvent faire l'objet d'approbation ou de contestation. (Emmanuel Picavet et Caroline GuibetLafaye, 11) indiquent à ce propos que :

" Dorénavant, la présence et l'implication de personnes non spécialistes sont largement perçues comme des éléments de précaution face à l'incertitude, et spécialement face au type d'incertitude qu'introduisent (ou accentuent) les développements des techniques. On estime, typiquement, qu'il serait imprudent de s'en remettre d'une manière par trop exclusive aux seuls spécialistes. En 
particulier, pour les raisons suivantes, bien exprimées par exemple en France à

l'occasion des «États généraux de la bioéthique » en 2009.» jouer un rôle déterminant dans la gestion des affaires publiques. Pour John Stuart Mill, ces droits ou libertés jouent deux rôles : ils favorisent l'amélioration d'une pensée déjà admise individuellement ou collectivement ainsi que la rectification d'une pensée erronée. Dans ces conditions, une bonne opinion peut être améliorée et une fausse opinion peut être rectifiée sous le coup de la discussion. En réalité, lorsqu'on permet à un individu de s'exprimer sur un sujet donné, il a l'opportunité d'améliorer son opinion ou de rectifier l'opinion fausse qu'il a de ce sujet. (John Stuart Mill, 91) écrit : «[...] s'habituer à corriger et compléter systématiquement son opinion en la comparant à celle des autres est la seule garantie qui la rende digne de confiance. » Or, en pratique, pour qu'un individu puisse comparer son opinion à celle des autres, il faudrait qu'il ait connaissance des opinions de ces derniers. Ce qui n'est possible que dans un cadre de discussion inclusive comme l'indique John Rawls (2009) à propos de la détermination des «principes de justice». Nous pouvons souligner dans cette perspective que si une maquette de la ville intelligente est présentée aux habitants, elle pourrait faire l'objet d'amélioration et d'adaptation à leurs réalités sociales. Le débat précédant la conception et la construction de la ville intelligente offre donc des possibilités de choix aux habitants. Ces derniers peuvent approuver ou désapprouver une maquette de la ville intelligente. Il s'agit, en réalité, d'une marque d'autonomie des habitants.

En somme, le présent travail part de l'idée que la ville intelligente favorise la participation citoyenne dans les collectivités publiques. Cependant, elle n'est pas construite sur la base de la technologie participative. Estimant à cet effet que les habitants ne sont pas associés au débat qui précède la construction de la ville intelligente, nous avons préconisé la mise en place de dispositifs et procédures susceptibles de favoriser la participation citoyenne dans la conception, la construction et la gestion de ladite ville afin qu'elle réponde mieux aux attentes des habitants. Un débat inclusif entre experts, spécialistes et les habitants peut donc permettre de marier la rationalité technologique avec les réalités sociales afin de rendre la ville intelligente plus intelligente. 


\section{BIBLIOGRAPHIE}

Authier J-Y., BacquéA M-H., Guerin-Pace F. (dir.), (2007), Le quartier. Enjeux scientifiques, actions politiques et pratiques sociales, Paris, La Découverte.

Bacqué M-H., Sintomer Y., (2001), « Gestion de proximité et démocratie participative », Les Annales de la Recherche Urbaine, $\mathrm{n}^{\circ}$ 90, p. 148-155.

Beaud O., Blanquer J-M. (dir.), (1999), La responsabilité des gouvernants, Paris, Descartes et Cie.

Bonnafous-Boucher M., Rendtorff J. D., (2013), La théorie des parties prenantes, Paris, La Découverte. Bouinot J., (2003), La ville intelligente, Paris, LGDJ.

Carrel M., (2013), « La gouvernance est-elle démocratique ? Les enjeux de la participation citoyenne », Informations sociales, vol. 179, nº 5, p. 144-151.

Damon J., « Une ville intelligente favorise le bien-être de ses habitants », Le Point, Paris, № 2303.

Deglise F., (2016), « Chronique - L'intelligence relative», Le Devoir Société. https:// www.ledevoir.com/opinion/chroniques/473829/chroniquefd-l-intelligence-relative Dewey J., (2005), Le public et ses problèmes. Traduit de l'anglais par J. Zask, Paris, Gallimard. Djemili A., Abbaoui M., (2011), « Démocratie participative et dynamique de la cité numérique en Algérie : le point de vue de l'architecte ». Revue Perspectives et Sociétés, Vol 2, n¹, p. 64-83.

Genro T., Ubiratan de S., (1998), Quand les habitants gèrent vraiment leur ville, Paris, Éditions Charles Leopold Mayer.

Georges B., (2016), «Big data Les nouveaux enjeux des données urbaines », Les Echos. https:// www.lesechos.fr/2016/06/les-nouveaux-enjeux-des-donnees-urbaines-228141

Lancon G., Buchoud N., (2003), Ces banlieues qui nous font peur. Une stratégie d'action pour transformer la gestion des quartiers d'habitat social, Paris, L'Harmattan.

Hugues M., (2008), Conseil de quartier. Démocratie de proximité : mode d'emploi, Paris, Éditions Territorial.

Maisl H., (1996), Le droit des données publiques, Paris, LGDJ.

Ogien A., Laugier S., (2014), Le principe démocratie. Enquête sur les nouvelles formes du politique, Paris, La Découverte.

Picavet E., (1996), Choix rationnel et vie publique, Fondement de la politique, Paris, PUF.

Picavet E., Guibet-Lafaye C., (2012), « La précaution, l'éthique et la structure de l'action ». Revue de Métaphysique et de Morale, PUF, p. 593-609.

Rawls J., (2009), Théorie de la justice, traduit de l'anglais (États-Unis) par Cathérine Audard, Paris, Éditions Points.

Rosanvallon P., (2015), Le bon gouvernement, Paris, Seuil.

Schöffer N., (1974), La Nouvelle Charte de la ville, Paris, Denoël/Gonthier.

Weil S., (1949), L'enracinement. Prélude à une déclaration des devoirs envers l'être humain, Paris, Gallimard. 


\section{NOTES}

1. En français : ouverture des données.

2. B. Georges, «Big data Les nouveaux enjeux des données urbaines », Les Echos, Juin 2016. [En

ligne] : https://www.lesechos.fr/2016/06/les-nouveaux-enjeux-des-donnees-urbaines-228141, consulté, le 30/12/2018.

3. ICF est un organisme à but non lucratif, situé à New York, qui étudie le développement des villes du XXI ${ }^{\mathrm{e}}$ siècle.

4. Les différents secteurs de fonctionnement qui composent l'espace urbain sont connectés entre eux.

5. Elle reste connectée avec les autres villes ou le reste du monde extérieur au sein duquel elle constitue une partie

6. Voir Aristote, (1891), Constitution d'Athènes, traduit par B. Haussoulier, Paris, Emile Boullon.

7. Il s'agit de Solon, Dracon, Clisthène, Périclès, etc.

8. Voir A. Ogien et S. Laugier, (2014), Le principe démocratie. Enquête sur les nouvelles formes du politique, Paris, La Découverte.

9. Voir E. Picavet et C. Guibet Lafaye, 2012, «La précaution, l'éthique et la structure de l'action ». Revue de Métaphysique et de Morale, PUF, p. 593-609.

10. Nous nous référons à cette ville, car elle est la première ville intelligente construite dans le monde entier.

\section{RÉSUMÉS}

La ville intelligente constitue une solution fiable apportée à la vulnérabilité des citoyens dans les collectivités publiques. Elle permet à celles-ci d'améliorer les conditions de vie des citoyens en rapprochant les services publics de ces derniers. Elle favorise surtout la démocratie participative par le biais des Nouvelles Technologies de l'Information et de la Communication. Malgré tout, il y a lieu de relever ici le manque de participation des habitants au débat qui précède la conception et la construction de ladite ville, par conséquent leurs avis ne sont pas pris en compte. Nous suggérons, à cet effet, la mise en place d'un dispositif permettant d'associer les habitants au débat qui précède la conception et la construction de la ville intelligente.

The smart city is a reliable solution to the vulnerability of citizens in public communities. It allows them to improve the living conditions of citizens by bringing public services closer to them. Above all, it promotes participatory democracy through New information and communication technologies. Nevertheless, it is necessary to note here the lack of participation of the inhabitants in the debate which precedes the design and the construction of this city, consequently their opinions are not taken into account. To this end, we suggest the establishment of a mechanism to involve residents in the debate that precedes the design and construction of the smart city.

Die Smart City ist eine zuverlässige Lösung für die Gefährdung von Bürgern in öffentlichen Gemeinden. Es ermöglicht ihnen, die Lebensbedingungen der Bürger zu verbessern, indem sie ihnen die öffentlichen Dienste näher bringen. Sie fördert vor allem die partizipative Demokratie durch neue Informations- und Kommunikationstechnologien. Dennoch ist hier die mangelnde 
Beteiligung der Einwohner an der Debatte zu bemerken, die dem Entwurf und dem Bau dieser Stadt vorausgeht, weshalb ihre Meinungen nicht berücksichtigt werden. $\mathrm{Zu}$ diesem Zweck schlagen wir die Einrichtung eines Mechanismus vor, um die Bewohner in die Debatte einzubeziehen, die dem Entwurf und der Errichtung der intelligenten Stadt vorausgeht.

INDEX

Mots-clés : ville intelligente, participation citoyenne, collectivité publique.

Keywords : smart city, citizen participation, public collectivity.

Schlüsselwörter : Smart City, Bürgerbeteiligung, öffentliche Gemeinschaft

\section{AUTEUR}

\section{ADÈHÈ ESSOSSIMNA POKORE}

Université Paris 1 Panthéon-Sorbonne 